\title{
ASTHMA
}

\section{Association of consumption of products containing milk fat with reduced asthma risk in pre-school children: the PIAMA birth cohort study}

\author{
A H Wijga, H A Smit, M Kerkhof, J C de Jongste, J Gerritsen, H J Neijens, \\ H C Boshuizen, B Brunekreef
}

Thorax 2003;58:567-572

See end of article for authors' affiliations

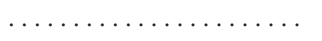

Correspondence to: Dr A Wijga, National Institute of Public Health and the Environment, Department of Chronic Disease Epidemiology (CZE), P O Box 1, 3720

BA Bilthoven, The Netherlands;

Alet.Wiiga@rivm.nl

Revised version received 20 February 2003

Accepted for publication

21 March 2003

\begin{abstract}
Background: Environment and lifestyle contribute to the development of asthma in children. Understanding the relevant factors in this relationship may provide methods of prevention. The role of diet in the development of asthma in pre-school children was investigated.

Methods: Data from 2978 children participating in a prospective birth cohort study were used. Food frequency data were collected at the age of 2 years and related to asthma symptoms reported at the age of 3 years.

Results: The prevalence of recent asthma at age 3 was lower in children who consumed (at age 2) full cream milk daily $(3.4 \%)$ than in those who did not $(5.6 \%)$ and in those who consumed butter daily $(1.5 \%)$ than in those who did not $(5.1 \%)$. The prevalence of recent wheeze was lower in children who consumed milk products daily $(13.7 \%)$ than in those who did not $(18.4 \%)$ and in children who consumed butter daily $(7.7 \%)$ than in those who did not (15.4\%). These effects remained in a logistic regression model including different foods and confounders (adjusted odds ratio $(\mathrm{Cl})$ for recent asthma: full cream milk daily $v$ rarely $0.59(0.40$ to 0.88$)$, butter daily $v$ rarely $0.28(0.09$ to 0.88$)$ ). Daily consumption of brown bread was also associated with lower rates of asthma and wheeze, whereas no associations were observed with the consumption of fruits, vegetables, margarine, and fish.

Conclusions: In pre-school children, frequent consumption of products containing milk fat is associated with a reduced risk of asthma symptoms.
\end{abstract}

t is widely believed that a modern Western lifestyle contributes to the risk of developing asthma. A number of aspects of lifestyle have changed over the past decades-including air pollution, the indoor environment, hygiene, and the burden of infections in early life-and their relation to asthma has been investigated. Diet is another aspect of lifestyle that has changed and the number of studies providing evidence on the relation between dietary factors and asthma has increased significantly in the last 10 years. Diet is one of the factors that has been hypothesised to play a role in the beneficial effects of an anthroposophic life style ${ }^{1}$ and of growing up in a farming environment. ${ }^{2}$

Reviews of the literature on nutrition and asthma have identified fruits, vitamin $\mathrm{C}$, vitamin $\mathrm{E}$, and beta-carotene as likely to be protective against asthma..$^{3-5}$ Different fatty acids, fish, and wholemeal bread may play a role, but further evidence is needed. ${ }^{3}$ Most of the studies included in these reviews have been on adults and therefore provide little evidence on nutritional factors that may influence the development of asthma early in childhood. A number of studies have been published on various dietary components and asthma (symptoms) in children. ${ }^{6-14}$ In addition, some of the recent studies on possible protective effects of growing up on a farm have reported on nutritional factors. ${ }^{215}{ }^{16}$ However, nearly all these studies were cross sectional. In the PIAMA (Prevention and Incidence of Asthma and Mite Allergy) birth cohort study we had the opportunity to investigate the relationship between food consumption and asthma symptoms in pre-school children followed prospectively.

\section{METHODS}

\section{Study population}

The PIAMA birth cohort study consisted of two parts: a placebo controlled intervention study in which the effect of mite impermeable mattress covers was studied and a natural history study in which no intervention took place. In this paper only data from the natural history part of the study are presented.

Pregnant women were recruited from the general population with the help of 52 midwife practices and obstetric clinics in three different regions of the Netherlands; 10232 pregnant women completed a validated short screening questionnaire on inhalant allergies. ${ }^{17}$ Women with any one of the following self-reported complaints-asthma, hay fever, house dust allergy, house dust mite allergy or pet allergywere defined as "allergic" $(\mathrm{n}=2949,29 \%)$. A random selection of 763 allergic women and 5084 non-allergic women were requested to join the natural history part of the study. Written informed consent was obtained from 3291 pregnant women (472 allergic (response 62\%) and 2819 non-allergic (response $55 \%)$ ). The participating children were born between July 1996 and October 1997.

\section{Data collection}

Data on respiratory and allergic symptoms and on potential risk factors were collected by postal questionnaires. Questionnaires, which included ISAAC (International Study of Asthma and Allergies in Childhood) core questions ${ }^{18}$ were sent to the parents during the last trimester of pregnancy, around 3 months after the birth of the child, and when the child was 1 , 2 and 3 years old.

In the study described here the association between nutrition and asthma was studied prospectively. Food consumption data collected at the age of 2 years were related to the prevalence of asthma symptoms reported at the age of 3 years. Around the child's second birthday, parents were asked to indicate from the following five options how often in the previous month the child had consumed a number of different 
Table 1 Characteristics of the study population

\begin{tabular}{llll}
\hline Characteristics & $\begin{array}{c}\text { Available } \\
\text { cases (n) }\end{array}$ & $\begin{array}{l}\text { Prevalences and } \\
\text { means in available } \\
\text { cases }\end{array}$ & $\begin{array}{l}\text { Prevalences and } \\
\text { means in imputed } \\
\text { dataset (n=2978) }\end{array}$ \\
\hline Sex (\% boys) & 2978 & 51.7 & 51.7 \\
Mean (SD) birth weight (g) & 2934 & $3517(536)$ & $3517(535)$ \\
First child (\%) & 2966 & 48.8 & 49.1 \\
Breast fed ever (\%) & 2936 & 81.9 & 81.9 \\
Breast fed for at least 8 weeks (\%) & 2911 & 61.2 & 61.0 \\
Smoking in the home (\%) & 2974 & 28.7 & 28.7 \\
Mother allergic (\%) & 2978 & 14.2 & 14.3 \\
Father allergic (\%) & 2977 & 29.1 & 29.0 \\
Mean (SD) mother's age (years) & 2933 & $30.4(3.8)$ & $30.4(3.8)$ \\
Mother's educational level*: & 2859 & & 23.4 \\
Low (\%) & & 23.2 & 42.3 \\
Intermediate (\%) & 42.2 & 34.3 \\
High (\%) & 2902 & 34.6 & 6.6 \\
Ever asthma (\%) & 6.3 & 14.8 \\
Recent asthma (\%) & 2898 & 4.6 & \\
Recent wheeze (\%) & 2894 & 14.6 & \\
\hline &
\end{tabular}

foods and drinks: not at all; a few times, but less than once a week; 1 or 2 days per week; $3-5$ days per week; 6 or 7 days per week.

Three outcome variables were defined based on data reported at the age of 3 years: "ever asthma", defined as ever having had a doctor's diagnosis of asthma; "recent asthma", defined as ever having had a doctor's diagnosis of asthma and having had asthma symptoms in the last 12 months and/or having used asthma medication in the last 12 months; and "recent wheeze", defined as having had one or more attacks of wheezing in the last 12 months. We are aware that a diagnosis of asthma is often uncertain in young children because of the lack of lung function measurements and other objective parameters that could distinguish between asthma and other respiratory complaints. At this age a reported doctor's diagnosis may therefore best be interpreted as the presence of asthma-like symptoms.

\section{Data analysis}

Food consumption data were available for 3042 children (92.4\% of the baseline population of 3291). To avoid reverse causation the data from 64 children were excluded from the analyses because their diet was apparently influenced by the child having an allergy to cow's milk. These children were defined as ever having had a doctor diagnosed food allergy and regularly using a cow's milk substitute. Data from 2978 children were therefore used for the analyses.

The five food consumption frequency categories from the questionnaire were summarised into three categories: rarely (defined as less than once a week), regularly (defined as on 1-5 days per week), and daily (defined as on 6 or 7 days per week).

Statistical analyses were performed with SAS version 8.1. Associations between asthma and wheeze and the consumption frequency of different foods were first examined univariately. Subsequently, foods that showed a significant association with asthma or wheeze incidence in this analysis were further examined using logistic regression analysis controlling for confounders. In the logistic regression analysis "daily" consumption and "regular" consumption were compared with the reference category "rare" consumption. If the prevalence of "rare" consumption was less than 5\% it was combined with the category "regularly" so that "daily" consumption was compared with consumption on 5 days per week or less.

Missing values were handled using multiple imputation. ${ }^{19}$ This method is recommended in order to avoid bias in analyses of epidemiological data with missing covariates. ${ }^{20}$ Imputation was carried out with MICE. ${ }^{21}$ In brief, five separate values for a missing data item were drawn from imputation models in which the value of this variable is predicted based on other variables in the dataset. We used linear imputation models

Table 2 Consumption frequenciest for different foods and prevalences of asthma and wheeze in children with daily consumption of these foods (weekly consumption in the case of fish) compared with all other children

\begin{tabular}{|c|c|c|c|c|c|c|c|c|c|}
\hline \multirow[t]{2}{*}{ Foods } & \multicolumn{3}{|c|}{ Consumption frequency } & \multicolumn{2}{|c|}{ "Ever asthma" (\%) } & \multicolumn{2}{|c|}{ Recent asthma (\%) } & \multicolumn{2}{|c|}{ Recent wheeze (\%) } \\
\hline & Rarely (\%) & Regularly (\%) & Daily (\%) & Daily users & Others & Daily users & Others & Daily users & Others \\
\hline Full cream milk & 61.8 & 6.1 & 32.1 & $4.6 * *$ & 7.5 & $3.4^{*}$ & 5.6 & 13.0 & 15.7 \\
\hline Semi-skim milk & 35.8 & 11.5 & 52.7 & 6.8 & 6.2 & 5.2 & 4.5 & 15.4 & 14.3 \\
\hline Milk products & 2.5 & 22.4 & 75.1 & $6.0 *$ & 8.2 & 4.6 & 5.7 & $13.7^{* *}$ & 18.4 \\
\hline White bread & 79.4 & 16.5 & 4.2 & $11.3^{*}$ & 6.3 & 7.8 & 4.8 & 18.4 & 14.7 \\
\hline Brown bread & 3.7 & 12.6 & 83.8 & $5.9 * *$ & 9.8 & $4.4^{*}$ & 7.2 & 14.6 & 16.2 \\
\hline Butter & 87.7 & 5.6 & 6.7 & $3.0 *$ & 6.8 & $1.5^{*}$ & 5.1 & $7.7^{* *}$ & 15.4 \\
\hline Margarine & 19.8 & 15.0 & 65.2 & 6.4 & 6.8 & 4.8 & 5.0 & 15.1 & 14.5 \\
\hline Cheese & 34.3 & 50.5 & 15.2 & 6.8 & 6.5 & 5.0 & 4.9 & 15.2 & 14.8 \\
\hline Fresh fruit & 3.8 & 29.8 & 66.4 & 6.6 & 6.4 & 4.8 & 5.0 & 14.9 & 14.8 \\
\hline Fruit juice & 20.3 & 40.0 & 39.6 & 5.9 & 6.9 & 4.3 & 5.2 & 14.6 & 15.0 \\
\hline Vegetables & 2.8 & 40.7 & 56.5 & 6.2 & 7.0 & 4.7 & 5.2 & 15.1 & 14.5 \\
\hline Meat & 3.8 & 38.6 & 57.6 & 6.5 & 6.6 & 5.0 & 4.8 & 15.1 & 14.5 \\
\hline Fish & 76.9 & 22.7 & 0.3 & 8.0 & 6.1 & 5.6 & 4.7 & 16.4 & 14.4 \\
\hline
\end{tabular}

${ }^{*} p<0.05,{ }^{*} p<0.01$, difference in asthma or wheeze prevalence between daily users and others.

†Consumption frequency: rarely=less than once a week; regularly= 1-5 days per week; daily $=6$ or 7 days per week. 
Table 3 Relationship between consumption frequency $†$ of full cream milk, milk products, butter and brown bread and prevalences of asthma and wheeze $(n=2978)$

\begin{tabular}{|c|c|c|c|}
\hline Regression models & "Ever asthma" ( $n=195)$ & Recent asthma $(n=145)$ & Recent wheeze $(n=442)$ \\
\hline \multicolumn{4}{|l|}{ Crude models } \\
\hline Full cream milk daily & $0.60(0.42 \text { to } 0.85)^{* *}$ & $0.59(0.40$ to 0.88$)$ * & 0.80 (0.64 to 1.00$)$ \\
\hline Full cream milk regularly & 0.99 (0.55 to 1.78$)$ & $0.79(0.38$ to 1.65$)$ & $0.91(0.59$ to 1.40$)$ \\
\hline Butter daily & $0.43(0.19$ to 0.97$)$ * & $0.28(0.09 \text { to } 0.88)^{*}$ & $0.46(0.27 \text { to } 0.79)^{*}$ * \\
\hline Butter regularly & $1.02(0.54$ to 1.91$)$ & $0.73(0.32$ to 1.67$)$ & $1.08(0.70$ to 1.66$)$ \\
\hline Milk products daily & $0.71(0.52$ to 0.98$)$ * & $0.81 \quad(0.55$ to 1.17$)$ & $0.70(0.56 \text { to } 0.88)^{* *}$ \\
\hline Brown bread daily & $0.58(0.41 \text { to } 0.82)^{* *}$ & $0.60(0.40 \text { to } 0.89)^{*}$ & 0.88 (0.67 to 1.16$)$ \\
\hline \multicolumn{4}{|l|}{ Adjusted model } \\
\hline Full cream milk daily & $0.54(0.34 \text { to } 0.88)^{*}$ & $0.53(0.30$ to 0.92$)$ * & $0.81 \quad(0.58$ to 1.13$)$ \\
\hline Full cream milk regularly & $0.83(0.44$ to 1.59$)$ & $0.73(0.33$ to 1.60$)$ & $0.87(0.54$ to 1.40$)$ \\
\hline Butter daily & $0.42(0.17$ to 1.01$)$ & $0.25(0.07 \text { to } 0.82)^{*}$ & $0.49(0.28$ to 0.87$)$ * \\
\hline Butter regularly & $0.97(0.50$ to 1.88$)$ & $0.73(0.31$ to 1.72$)$ & $1.12(0.71$ to 1.77$)$ \\
\hline Milk products daily & $0.74(0.53$ to 1.03$)$ & $0.82(0.55$ to 1.21$)$ & $0.68(0.54 \text { to } 0.86)^{* *}$ \\
\hline Brown bread daily & $0.60(0.41 \text { to } 0.87)^{* *}$ & $0.60(0.39$ to 0.93 )* & $1.0 \quad(0.74$ to 1.33$)$ \\
\hline Semi-skimmed milk daily & 0.83 (0.53 to 1.31$)$ & $0.75(0.45$ to 1.26$)$ & 0.99 (0.71 to 1.38$)$ \\
\hline Semi-skimmed milk regularly & $1.07(0.64$ to 1.80$)$ & $0.72(0.37$ to 1.38$)$ & $1.05(0.72$ to 1.52$)$ \\
\hline Margarine daily & $0.94 \quad(0.62$ to 1.42$)$ & $0.82(0.51$ to 1.31$)$ & $0.96(0.71$ to 1.29$)$ \\
\hline Margarine regularly & 1.03 (0.62 to 1.71$)$ & $0.87(0.48$ to 1.57$)$ & 0.96 (0.66 to 1.39$)$ \\
\hline Male sex & $2.20(1.59 \text { to } 3.03)^{* *}$ & $2.26(1.55 \text { to } 3.29)^{* *}$ & $1.52(1.23 \text { to } 1.88)^{* *}$ \\
\hline \multicolumn{4}{|c|}{ Birth weight (reference $<3000 \mathrm{~g}$ ) } \\
\hline $3000-3499 \mathrm{~g}$ & $0.73(0.44$ to 1.20$)$ & $0.60(0.34$ to 1.08$)$ & 0.77 (0.55 to 1.07$)$ \\
\hline $3500-3999 \mathrm{~g}$ & $0.90(0.55$ to 1.46$)$ & $0.82(0.48$ to 1.40$)$ & $0.76(0.54$ to 1.07$)$ \\
\hline$\geqslant 4000 \mathrm{~g}$ & $0.59(0.34$ to 1.03$)$ & $0.65(0.35$ to 1.19$)$ & $0.61(0.42$ to 0.90$)$ * \\
\hline Older sibling(s) present & $1.31(0.95$ to 1.80$)$ & $1.41(0.98$ to 2.04$)$ & $1.21(0.97$ to 1.51$)$ \\
\hline Father allergic & $1.58(1.16 \text { to } 2.16)^{* *}$ & $1.41 \quad(0.98$ to 2.02$)$ & $1.63(1.31 \text { to } 2.02)^{* *}$ \\
\hline Mother allergic & $1.81(1.26 \text { to } 2.61)^{* *}$ & $2.16(1.44 \text { to } 3.23)^{* *}$ & $1.30(0.98$ to 1.72$)$ \\
\hline \multicolumn{4}{|c|}{ Maternal education (reference low) } \\
\hline Intermediate & $1.23(0.81$ to 1.88$)$ & $1.29(0.79$ to 2.10$)$ & 1.10 (0.83 to 1.44$)$ \\
\hline High & $1.42(0.88$ to 2.28$)$ & $1.36(0.78$ to 2.36$)$ & $1.08(0.80$ to 1.46$)$ \\
\hline Breast fed $\geqslant 8$ weeks & $0.69(0.50$ to 0.95$)$ * & $0.63(0.44 \text { to } 0.91)^{*}$ & $0.62(0.49 \text { to } 0.77)^{* *}$ \\
\hline Smoking in the home & $0.83(0.59$ to 1.19$)$ & $0.80(0.53$ to 1.20$)$ & $1.12(0.88$ to 1.42$)$ \\
\hline
\end{tabular}

including all variables correlated more than 0.1 with the variable to be imputed, and used predictive mean matching to draw imputed values from this model. This drawing method takes as an imputed value the observed value of the child for which the prediction model predicts a value closest to the predicted value of the child with the missing value. The parameters of the imputation model are established using Gibbs sampling, and thus differ between each of the five imputations so that different matches are usually made. This method assures that imputed values follow the same (conditional) distribution as the observed values. The multiple imputation procedure yields five complete datasets in which each missing value is replaced by an imputed value which is usually different for each data set, while the observed data are always the same in each data set. With these five data sets, five different logistic regressions (or other analyses) were carried out and the five results were pooled using SAS PROC MIANALYSE in order to obtain a single final result. The percentage of missing values for individual foods included in the logistic regression model was $1.0 \%$ and the percentage of missing values for potential confounders included in the model ranged from $0 \%$ for the child's sex and maternal allergy to $4.0 \%$ for maternal education. $2.8 \%$ of the study population had missing values for the outcome variables. Without the imputation of missing values the total number of complete cases available for the logistic regression analysis was 2594 (87.1\% of the study population of 2978 children).

\section{RESULTS}

The characteristics of the study population for the available cases and for the imputed dataset are shown in table 1 . The prevalences of different characteristics were similar in the available cases and in the imputed dataset. Small differences were found for maternal education and for asthma symptoms, the latter because loss to follow up was higher in children who were reportedly symptomatic at the age of 2 years. At the age of 3 years the prevalence of "ever asthma" was $6.6 \%$, of "recent asthma" $4.9 \%$, and of "recent wheeze" $14.8 \%$ (table 1). Of the 442 recent wheezers, $28.4 \%$ had ever had a doctor's diagnosis of asthma and $26.6 \%$ had recent asthma.

Consumption frequencies of different foods are shown in table 2, together with the prevalences of asthma and wheeze in children who consume these foods daily (weekly in the case of fish) compared with all other children. The results shown are based on the imputed dataset but differ very little from the results based on available cases only (data not shown). In this crude analysis, daily consumption of full cream milk, milk products (including products like yoghurt and chocolate milk), butter, and brown bread were significantly associated with low rates of asthma and/or wheeze. Children with daily consumption of fruit juice and of vegetables had lower asthma rates than other children, but these differences were not statistically significant. Eating fish at least once a week was not associated with asthma or wheeze.

Associations were observed between the consumption frequencies of different foods included in the questionnaire. Some foods were apparently used as substitutes. Of the children with daily consumption of full cream milk, $83 \%$ rarely consumed semi-skimmed milk; of the children with daily consumption of brown bread, $90 \%$ rarely consumed white bread; and of the children with daily consumption of butter, $73 \%$ rarely consumed margarine. Positive associations existed between the consumption frequencies of foods that, in the Netherlands, are usually eaten together such as bread on the 
one hand and milk, margarine and cheese on the other. Associations were also present between the consumption frequencies of full cream milk and butter, and between the consumption frequencies of milk products and brown bread. The prevalence of daily butter consumption was $10 \%$ in the children with a daily consumption of full cream milk and 5\% in the other children. The prevalence of daily consumption of milk products was $78 \%$ in the children with daily consumption of brown bread and $63 \%$ in the other children. Despite the associations between the consumption frequencies of different foods, a logistic regression model including full cream milk, milk products, butter, and brown bread together showed similar associations between the consumption frequencies of these foods and asthma and wheeze as the crude analyses (data not shown). Of the other foods covered in the questionnaire, only semi-skimmed milk and margarine were found to influence the effect estimates of full cream milk, milk products, butter, and brown bread and were included in the model (table 3).

Whereas there was some overlap between the groups of children with daily consumption of full cream milk, milk products, butter and brown bread, the consumption frequencies for different foods were associated with different background variables. Daily consumption of full cream milk was more prevalent in firstborn children than in others $(38 \%$ $v 26 \%)$ and was inversely related to the child's birth weight (40\% for birth weight $<3000 \mathrm{~g} v 30 \%$ for birth weight $>3500 \mathrm{~g}$ ). Daily consumption of butter was associated with the mother's educational level (3.6\% for low level of education $v 8.8 \%$ for high level of education). Daily consumption of milk products and daily consumption of brown bread were both positively related to the mother's level of education (milk products: $68 \%$ for low level of education $v 76 \%$ for high level of education; brown bread: $75 \%$ for low level of education $v 88 \%$ for high level of education) and negatively related to the prevalence of smoking in the child's home (milk products: $65 \%$ in smoking families $v 71 \%$ in non-smoking families; brown bread: $71 \%$ in smoking families $v 78 \%$ in non-smoking families).

In the final regression model the following variables were included in addition to the nutritional variables: sex, birth weight, presence of older siblings (yes/no), parental allergy (having (had) asthma or having an inhalant allergy), maternal level of education, breast feeding for at least 8 weeks (yes/no), and smoking in the home when the child was 2 years old (at least once a week $v$ less than once a week). Effects of region, maternal smoking during pregnancy, and parental asthma were also assessed but these variables were not included in the final model. In this model odds ratios significantly lower than 1 were again observed for the relationships between daily consumption of full cream milk, milk products, butter, and brown bread and asthma and/or wheeze. When the same analyses were carried out using only cases with complete data $(n=2594)$ the results were similar, but brown bread was not significantly associated with recent asthma and its association with "ever asthma" lost statistical significance in the adjusted regression model. Consumption frequencies of fish, fruits, and vegetables were not associated with asthma symptoms in the adjusted regression analysis. Besides the nutritional factors, maternal allergy, paternal allergy, being a boy, and not having been breast fed for at least 8 weeks were significantly associated with both asthma and wheeze in the adjusted regression model. Low birth weight was significantly associated with wheeze. The estimates for the effects of full cream milk, milk products, butter, and brown bread on asthma and wheeze were of the same order of magnitude as the effects of the established risk factors parental allergy and being a boy. ${ }^{22}$

\section{DISCUSSION}

There was consistent evidence for an inverse relationship between the consumption frequency of products containing milk fat on the one hand and asthma and wheeze in pre-school children on the other. An inverse association was also observed between the daily consumption of brown bread and asthma.

We considered the possibility that our results were influenced by methodological aspects of the study. Of the 5847 pregnant women invited to join the natural history part of the study, 3291 (56\%) agreed to do so. Of this study population of 3291 at baseline, 3141 (95.4\%) were still in the cohort when the children were 3 years old and food consumption data were available for 3042 children. We have no data on the women who were invited but did not join the study, but comparison of the PIAMA population with data available on the general Dutch population suggests that, in the PIAMA population, parents with only primary education and smoking parents may be underrepresented. The adjusted regression analysis did not indicate, however, that the associations observed were influenced by parental education or smoking.

Although standard methods (ISAAC) were used to assess the prevalence of asthma and wheeze, misclassification may have occurred because the diagnosis of asthma is often uncertain in children aged 3 years or younger. However, as there is no reason to assume that parents of children with high intakes of milk fat would report asthma symptoms and diagnoses differently from other parents, differential misclassification is unlikely. Misclassification independent of exposure, if it occurred, would result in dilution of the associations observed.

To avoid finding associations due to reverse causation, 64 children with cow's milk allergy were excluded from the analyses. Children with cow's milk allergy generally avoid cow's milk and are more likely than other children to develop further allergic complaints and asthma. We investigated whether consumption of specific foods influences the risk of developing asthma symptoms and therefore excluded children whose illness (cow's milk allergy with the associated risk of developing asthma) is the cause rather than the result of their food consumption habits. Of the children who were included in the analyses, only $27(<1 \%)$ did not use milk and had not used milk products in the previous month. Parental allergy was more prevalent in these 27 children than in the other children, so we cannot exclude the possibility that, in at least some cases, their own allergy may have induced parents not to give their child cow's milk. Inclusion of these children may have introduced inverse causation, but their exclusion could have hidden an existing association. The number of children involved, however, is so small that their exclusion would not have influenced our results.

Food consumption was assessed using a short and simple food frequency questionnaire. From the different methods available to measure food consumption, the food frequency questionnaire is considered the most appropriate for epidemiological studies and has been validated in a large number and variety of studies and populations. ${ }^{23}$ Our questionnaire was short and simple, asking about only a limited number of broad food groups and not about, for example, portion sizes. Our data enable us to rank children according to their consumption frequencies of a number of food groups, but cannot be used to calculate total energy or nutrient intakes. We have assumed that parents can correctly report whether their child eats, for example, brown or white bread every day, and that we can thus discriminate between white and brown bread consumers. The questionnaire cannot, however, identify small differences in intakes between subjects and differences that result from portion sizes rather than from consumption frequency. We are therefore confident that the associations observed are real and not the result of invalid measurements, but we cannot exclude the possibility that other existing associations between food consumption and asthma symptoms have not been detected.

Nutrition is known to be related to other aspects of lifestyle. Foods that are found to be beneficially related to specific 
health outcomes may be indicators of an unidentified aspect of a healthy lifestyle rather than being protective themselves. For foods with a high content of milk fat we think this unlikely to be the case, however, because these are generally not perceived as typical constituents of a healthy diet and lifestyle.

The foods that were associated with asthma and/or wheeze in our study population-in particular milk (products) and bread-are major components of the diet of 2 year old children. According to a Dutch national food consumption survey conducted in 1998, ${ }^{24}$ children aged 1-4 years derived, on average, $22 \%$ of their total energy intake from milk and milk products and $12 \%$ from bread. We did not find associations between the consumption frequencies of fruits and vegetables or fish, on the one hand, and asthma and wheeze on the other. This may be due to inadequate identification by our questionnaire of children with high and low intakes of these foods. With respect to fruits and vegetables, another explanation could be that the role of antioxidant vitamins is age dependent and less important in young children than in adults, particularly in adult smokers. The observed inverse association between daily consumption of brown bread and asthma is in line with results of a multicentre study of middle aged men in which bread consumption was found to be beneficially associated with pulmonary function. ${ }^{25}$ The association could be related to antioxidant components of whole grains. ${ }^{26}$ We cannot exclude the possibility, however, that the choice of brown bread over white bread is an indicator of a healthy lifestyle and that other unidentified aspects of lifestyle could (partly) explain the observed association between brown bread and asthma.

Associations between consumption of milk and asthma or other respiratory or allergic symptoms in children have also been observed in other studies. These were cross sectional studies that focused either on nutrition and asthma or on the effects of growing up in a farming environment. Von Ehrenstein et al $^{15}$ observed an association between consumption of full cream milk, but not of semi-skimmed milk, and a lower prevalence of asthma and hay fever in German children aged 5-7 years. Hijazi et al reported inverse linear relationships between intakes of milk and vegetables and having had asthma and wheeze in the past 12 months in 12 year old children in Saudi Arabia. In the ALEX study ${ }^{2}$ associations between consumption of farm milk in the first year of life and lower frequencies of asthma, hay fever, and atopic sensitisation at the age of 6-13 years were observed in rural areas of Austria, Germany, and Switzerland. In Greece an association was observed between consumption of farm milk under the age of 5 years and lower prevalence of atopy in children aged 11-19 years. ${ }^{16}$ Part of the evidence on an association between milk consumption and asthma comes from studies on traditional farm life and atopy and asthma. From preliminary data currently being collected and from home visits, we know that fewer than $2 \%$ of the children in our study live on farms. In the regression analysis a variable for the region where the children were recruited had no effect on the associations observed and was left out of the final model. We therefore consider it unlikely that in our study the association between consumption of full cream milk and asthma symptoms is the result of an underlying association between farm life and asthma symptoms.

Associations between butter consumption and asthma or atopy have been observed in a number of studies that focused mainly on fatty acids and atopy. Von Mutius et $a l^{27}$ in their study on time trends in the prevalence of asthma and allergic diseases in eastern Germany found an inverse association between changes in the consumption of butter and hay fever and atopic sensitisation. Haby et al $^{8}$ found that a high intake of polyunsaturated fats (PUFAs) increased the risk of having asthma in Australian children aged 3-5 years. A high intake of PUFAs was defined as usually using them on bread and in cooked meals, while the control group consisted of children who usually used saturated fats including butter and/or monounsaturated fats. Bolte et $a l^{13}$ found an association between margarine consumption (compared with butter consumption) and allergic sensitisation and rhinitis symptoms in German children aged 5-14 years, but no association with hay fever and asthma. Dunder et $\mathrm{l}^{14}$ studied Finnish children aged 3-18 years at baseline and found, both cross sectionally and after 9 years of follow up, that children with atopic disease had consumed less butter than those without atopic disease.

Our data provide evidence for a beneficial association between daily consumption of milk fat containing products and asthma and wheeze in young children. Various components of the products involved could play a role, such as different fatty acids, but also antioxidants or other micronutrients. Several authors have hypothesised that the increase in the prevalence of asthma, eczema, and allergic rhinitis in the western world is related to the reduction in the consumption of saturated fat and the increase in the consumption of n-6 polyunsaturated fatty acids and, in particular, linoleic acid. ${ }^{28}{ }^{29}$ We cannot estimate from our data the children's total intakes of different types of fat. The choice for full cream milk over semi-skimmed milk and for butter over margarine is very likely to result in a higher proportion of saturated fat and thus a lower proportion of unsaturated fat in the diet. If the proportion of linoleic acid in the diet is relevant to the development of asthma, this could partly explain our findings. In our data we did not observe an independent association between margarine consumption and asthma or wheeze, however, so we have no direct evidence for an unfavourable effect of linoleic acid.

We conclude that our results provide evidence for a relationship between nutrition and asthma. As a hypothesis for further research we suggest that the foods and nutrients that are relevant in this relationship may be specific for different age groups or other subgroups depending, among other factors, on the quantitative importance of different foods in the diet. In our prospective study we found evidence for a risk reducing effect of products containing milk fat on the development of asthma and wheeze in pre-school children.

Authors' affiliations

A H Wiiga, H A Smit, H C Boshuizen, National Institute of Public Health and the Environment, Department of Chronic Disease Epidemiology (CZE), Bilthoven, The Netherlands

M Kerkhof, J Gerritsen, Department of Paediatric Respiratory Medicine, University Hospital Groningen, Groningen, The Netherlands

M Kerkhof, Department of Epidemiology and Statistics, University of Groningen, Groningen, The Netherlands

J C de Jongste, H J Neiiens, Department of Paediatrics, Erasmus University Medical Center/Sophia Children's Hospital, Rotterdam, The Netherlands

B Brunekreef, Institute for Risk Assessment Sciences, University of Utrecht, Utrecht, The Netherlands

Funded by National Institute of Public Health and the Environment, Bilthoven.

\section{REFERENCES}

1 Alm JS, Swartz J, Lilia $G$ et al. Atopy in children of families with an anthroposophic lifestyle. Lancet 1999;353:1485-8.

2 Riedler J, Braun-Fahrländer C, Eder W, et al. Exposure to farming in early life and development of asthma and allergy: a cross-sectional survey. Lancet 2001;358:1129-33.

3 Smit HA. Chronic obstructive pulmonary disease, asthma and protective effects of food intake: from hypothesis to evidence? Respir Res $2001 ; 2: 261-4$

4 Godden DJ, Devereux GS, Anderson WJ. Environmental lung disease: the role of diet. Monaldi Arch Chest Dis 1999;54:479-84.

5 Fogarty A, Britton J. The role of diet in the aetiology of asthma. Clin Exp Allergy 2000;30:615-27.

6 Hodge L, Salome CM, Peat JK, et al. Consumption of oily fish and childhood asthma risk. Med J Aust 1996;1 64:137-40

7 Hijazi N, Abalkhail B, Seaton A. Diet and childhood asthma in a society in transition: a study in urban and rural Saudi Arabia. Thorax 2000;55:775-9. 
8 Haby MM, Peat JK, Marks GB, et al. Asthma in preschool children: prevalence and risk factors. Thorax 2001;56:589-95.

9 Huang SL, Lin KC, Pan WH. Dietary factors associated with physician-diagnosed asthma and allergic rhinitis in teenagers: analyses of the first Nutrition and Health Survey in Taiwan. Clin Exp Allergy 2001;31:259-64.

10 Takemura Y, SakuraiY, Satoshi H, et al. The relationship between fish intake and the prevalence of asthma: the Tokorozawa Childhood Asthma and Pollinosis Study. Prevent Med 2002;34:221-5.

11 Forastiere F, Pistelli R, Sestini $P$, et. Consumption of fresh fruit rich in vitamin $C$ and wheezing symptoms in children. Thorax 2000;55:283-8.

12 Cook DG, Carey IM, Whincup PH, et al. Effect of fresh fruit consumption on lung function and wheeze in children. Thorax 1997;52:628-33.

13 Bolte G, Frye C, Hoelscher B, et al. Margarine consumption and allergy in children. Am J Respir Crit Care Med 2001;163:277-9.

14 Dunder T, Kuikka L, Turtinen J, et al. Diet, serum fatty acids and atopic disease in childhood. Allergy 2001;56;425-8.

15 Von Ehrenstein OS, von Mutius E, Illi S, et al. Reduced hay fever and asthma among children of farmers. Clin Exp Allergy 2000;30:187-93.

16 Barnes M, Cullinan P, Athanasaki $P$, et al. Crete: does farming explain urban and rural differences in atopy? Clin Exp Allergy 2001;31:1822-8.

17 Lakwijk N, van Strien RT, Doekes G, et al. Validation of a screening questionnaire for atopy with serum lgE tests in a population of pregnant Dutch women. Clin Exp Allergy 1998;28:454-8.

18 The International Study of Asthma and Allergies in Childhood (ISAAC) Steering Committee. Worldwide variation in prevalence of symptoms of asthma, allergic rhinocunjunctivitis and atopic eczema: ISAAC. Lancet 1998:351:1225-32.
19 Rubin D.B. Multiple imputation for nonresponse in surveys. New York: Wiley, 1987

20 Greenland S, Finkle WD. A critical look at methods for handling missing covariates in epidemiologic regression analyses. Am J Epidemiol 1995: 142:1255-64

21 Van Buuren S. Oudshoorn CGM. Multivariate imputation by chained equations. MICE version 1.0 user's manual. TNO report
PG/GZ/00.038. Leiden: TNO Prevention and Health, 2000.

22 Morgan WJ, Martinez FD. Risk factors for developing wheezing and asthma in childhood. Pediatr Clin North Am 1992;39:1 185-203.

23 Willet W, Lenart E. Reproducibility and validity of food-frequency questionnaires. In: Willet W, ed. Nutritional epidemiology. 2nd ed. Oxford: Oxford University Press, 1998: 101-47.

24 Hulsh of KFAM, Kistemaker C, Kruizinga AG. De bijdrage van groepen voedingsmiddelen aan de inname van energie en voedingsstoffen Voedselconsumptiepeiling 1997-1998. TNO rapport V98.807. Zeist 1998.

25 Tabak C, Smit HA, Räsänen L, et al. Dietary factors and pulmonary function: a cross-sectional study in middle aged men from three European countries. Thorax 1999:54:1021-6.

26 Thompson LU. Antioxidants and hormone-mediated health effects of whole grains. Crit Rev Food Sci Nutr 1994;34:473-97.

27 Von Mutius E, Weiland SK, Fritzsch C, et al. Increasing prevalence of hay fever and atopy among children in Leipzig, East Germany. Lancet 1998:351:862-6.

28 Hodge L, Peat JK, Salome C. Increased consumption of polyunsaturated oil may be a cause of increased prevalence of childhood asthma. Aust NZ J Med 1994;24:727

29 Black PN, Sharpe S. Dietary fat and asthma: is there a connection? Eur Respir J 1997;10:6-12.

\section{LUNG ALERT}

\section{Influenza vaccination reduces risk of hospitalisation for heart disease and CVD in subjects aged $\geqslant 65$ years}

$\Delta$ Nichol KL, Nordin J, Mullooly J, et al. Influenza vaccination and reduction in hospitalisations for cardiac disease and stroke among the elderly. N Engl J Med 2003;348: 1322-32

erious influenza related illnesses cause considerable morbidity and mortality to patients and put enormous pressure on health, social care and other services. An increased risk of ischaemic heart disease and cerebrovascular disease (CVD) are known to be associated with upper respiratory tract illnesses. In UK studies a substantial reduction in complications, hospital admissions, and mortality has been described in targeted subjects who had received influenza vaccine compared with matched controls who did not have vaccination. Nichol et al have conducted an observational study on two large cohorts of subjects aged 65 or above during the 1998-9 and 1999-2000 influenza seasons. Each cohort consisted of over 140000 subjects. Clinical data for those who received influenza vaccination were compared with those who did not receive vaccination. Baseline demographic and health characteristics of the subjects were controlled before comparisons were made between the two groups. The authors found that subjects who received influenza vaccination had clinically and statistically significant reductions in the risks of hospitalisation for heart disease, CVD, pneumonia, or influenza and death from all causes.

This study provides further evidence of the benefits of influenza vaccination in the elderly population and lends support to the current UK policy of recommending influenza vaccination to all subjects aged 65 or over. 